\title{
Extension of the FundamentalWave Library towards Multi Phase Electric Machine Models
}

\author{
Christian Kral \\ Electric Machines, Drives and Systems \\ 1060 Vienna, Austria \\ dr.christian.kral@gmail.com
}

\author{
Anton Haumer \\ Technical Consulting \\ 3423 St.Andrä-Wördern, Austria \\ a.haumer@haumer.at
}

\author{
Reinhard Wöhrnschimmel \\ AIT GmbH \\ 1210 Vienna, Austria
}

\begin{abstract}
Electric machine theory and electric machine simulations models are often limited to three phases. Up to the Modelica Standard Libray (MSL) version 3.2 the provided machine models were limited to three phases. Particularly for large industrial drives and for redundancy reasons in electric vehicles and aircrafts multi phase electric machines are demanded. In the MSL 3.2.1 an extension of the existing FundamentalWave library has been performed to cope with phase numbers greater than or equal to three. The developed machine models are fully incorporating the multi phase electric, magnetic, rotational and thermal domain. In this publication the theoretical background of the machines models, Modelica implementation details, the parametrization of the models and simulation examples are presented.
\end{abstract}

Keywords: Modelica Standard Library, multi phase, electric machine models, induction machine, synchronous machine, synchronous reluctance machine

\section{Introduction}

Three phase induction, synchronous and synchronous reluctance machines are state of the art solutions for industrial applications, traction drives in electric vehicles, railways, trams, and underground trains, as well as air craft motors and generators. If a full leg of the supplying three phase converter fails, the machine cannot be operated any more, once stopped. In particular, the higher ambient temperatures of traction machines may cause the power electronics to fail. In order to overcome machine outage due to a single converter leg failure, phase numbers greater than three may be used for electric machines and power electronics. In the following multi phase will indicate phase numbers greater than three. If it is referred to only three phases, this will be indicated explicitly.

Multi phase drives consist of the multi phase machine including an inverter with power electronics plus control. A phase number greater than three thus requires a higher number of power electronic switches, such as IGBTs or MOSFETs, etc. The higher phase numbers, however, increase cost and add complexity to the drive structure.

However, phase numbers equal to $2^{n}$ with integer $n$ are excluded from the actual implementation. The reason for excluding these phase numbers is that for example four or eight phase machines have to be handled differently since two phase are separated by $\pi / 2$, not $\pi$. In general, two different philosophies of multi phase drives exist:

First, the number of phases is divisible by two. In industry, typically, six phase machines are used to overcome maximum power limitations of power electronics supplying high power machines in the Megawatt range [1]. In this case maximum power of power electronics is doubled by using two three phase converters supplying a six phase machine. Usually, the phase winding orientations of the two three phase are spatially shifted by $30^{\circ}$ in order to additionally reduce the magnitudes of space harmonics caused by the winding magneto motive force (MMF) and to reduce the torque ripple of the machine, respectively. The implementation of a six phase winding does not significantly increase cost of the electric machine compared with a three phase machine with the same power. Solely the additional winding ends have to be conducted to the terminal box. For six phase drives, the cost of power electronics increases due the double number of power electronic switches for the additional legs. For doubling the power of industrial drives the double cost of power electronics is in line with doubling the power. However, for traction machines six or nine phase machines are used for redundancy reasons $[2,3]$. In this case several state of the art three phase converters can be used. The redundancy concept is then realized with standard components which is cheaper than designing the individual legs of the inverter. Yet, several three phase inverters of smaller power rating are usually more expensive than a three phase inverter of the same total power. Due to the multiple three phase inverters installation space increases, too. Yet, state of the art control for three phase drives can be adapted with relatively low effort due to modularly using three phase converters.

Second, the number of phases is not divisible by two. In this case mostly five (or seven) phase drives are used [4-6]. The drawback over a six phase inverter is that the modularity of the power electronics design is lower and thus cost may be higher and more design space may be required. 
For the sake of completeness one more redundancy concept will be discussed here, even though it is not related with multi phase electric machines. Redundant drives with three phase machines may use modified topologies which either use an additional leg or additional switches to operate the machine in case of a failure. These topologies have the capability to be reconfigured when a failure occurs [7,8]. Depending on the actual topology even the full power rating may be provided to the electric machine.

For controlling multi phase electric drives it is advantageous to control current components which represent the fundamental wave MMF and magnetic voltage, respectively [9]. The pulse width modulation scheme for multi phase converters with phase numbers not divisible by three has to be adapted so that a symmetrical supply can be achieved. A technical paper dealing in more detail with analysis of the multi phase drive control is also submitted to the Modelica 2014 conference and will be cited properly, in case it gets accepted.

In Modelica the first three phase electric machine models have been introduced with the MSL 2.1 in 2004 [10, 11]. An alternative implementation with magnetic fundamental wave phasors was introduced in MSL 3.2 in 2010 [12]. Since then in these models copper loss, (eddy current) core loss, friction loss, stray load loss, PM loss and brush loss are taken into account. Multi phase electric machine models have already been published decades ago $[13,14]$. Yet, in most computer simulations tools there are currently only three phase machine models available. However, in the MSL 3.2.1 version of the package Modelica.Magnetic.FundamentalWave new multi phase electric machine models are introduced. In general, arbitrary phase numbers for stator (and rotor) windings may be used - excluding phase numbers equal to $2^{n}$ with integer $n$. This article provides the theoretical background, details about the implementation, parametrization schemes and some examples.

\section{Fundamental Wave Theory}

Multi phase electric machine theory often relies on phasor transformations of currents, voltages and magnetic fluxes $[15,16]$. A typical transformation is the symmetrical components of the instantaneous values. In case of fully symmetrical supply the machine equations based on the symmetrical components of instantaneous components can be simplified extensively.

The FundamentalWave machine models only consider fundamental wave effects so there is also a complex phasor representation of the fundamental wave of the magnetic flux and magnetic potential (difference), respectively. The connector definition of the FundamentalWave library shows:

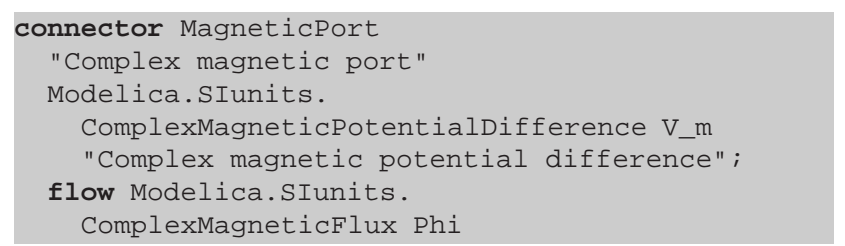

"Complex magnetic flux";

end MagneticPort;

Please note, that the potential and flow variable of the connector represent instantaneous quantities $\underline{\Phi}=\Phi_{\text {re }}+\mathrm{j} \Phi_{\text {im }}$ and $\underline{V}_{m}=V_{m, \text { re }}+\mathrm{j} V_{m, \text { im }}$. The complex magnetic quantities represent a spatial distribution of magnetic flux and magnetic potential (difference):

$$
\begin{aligned}
\Phi(\varphi) & =\operatorname{Re}\left[\left(\Phi_{\mathrm{re}}+\mathrm{j} \Phi_{\mathrm{im}}\right) e^{-\mathrm{j} \varphi}\right] \\
& =\Phi_{\mathrm{re}} \cos (\varphi)+\Phi_{\mathrm{im}} \sin (\varphi) \\
V_{m}(\varphi) & =\operatorname{Re}\left[\left(V_{m, \mathrm{re}}+\mathrm{j} V_{m, \mathrm{im}}\right) e^{-\mathrm{j} \varphi}\right] \\
& =V_{m, \mathrm{re}} \cos (\varphi)+V_{m, \mathrm{im}} \sin (\varphi)
\end{aligned}
$$

The complex potential (difference) $\underline{V}_{m}$ introduced in the connector definition represents the total magnetic potential (difference) of all poles. This quantity can, thus, also be seen as the complex magnetic potential difference of an equivalent two pole machine. Physical interpretations of the complex magnetic phasors are presented and discussed in [12].

Voltages and currents are instantaneous quantities, so arbitrary waveforms and operating conditions are covered. Therefore, the machines can also be supplied with asymmetric voltages or currents. It is yet assumed that only fundamental wave effects due to these asymmetries are considered. Supply voltage or current imbalance give rise to time transient magnitudes of the magnetic flux and magnetic potential (difference). Each of the spatial field distributions can be interpreted as a forward and backward traveling fundamental wave component. Those effect of the two waves is correctly taken in account by the proposed approach.

Particular supply imbalances and certain asymmetries may cause magnetic flux and magnetic potential (difference) phasors which are not related with the fundamental wave. Those higher harmonic waves are not covered by the FundamentalWave library. It is therefore the user's responsibility to consider these model limitations - with particular focus on the supply conditions.

Any higher harmonic wave effects are also not taken into account by the FundamentalWave library. In case of higher harmonic waves an alternative implementation has to be considered as presented in [17]. The impact of rotor saliency on the fundamental wave components of magnetic flux and magnetic potential (difference) is, however, considered in the presented implementation.

Concentrated windings and fractional slot windings, respectively, are very common in PM synchronous machines due to better field weakening capabilities, higher pole numbers and higher power density. Such fractional slot windings can be considered in the FundamentalWave library, as long as the main power exchanging harmonic component is interpreted as fundamental wave. All higher harmonic waves cause by fractional slot windings are not explicitly considered, but the total effect of those higher harmonics can be taken into account by the total leakage inductance of the stator winding. 


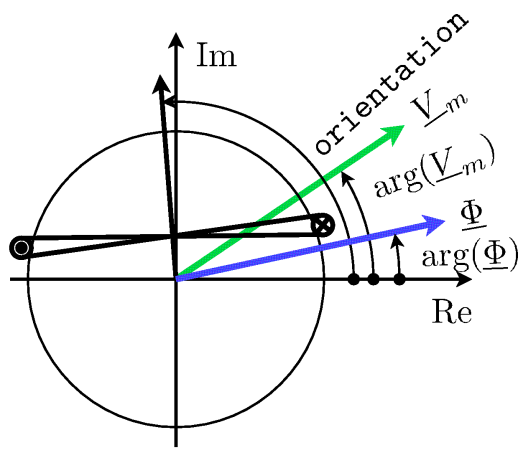

Figure 1: Coil of an equivalent two pole machine with orientation; complex magnetic potential (difference) phasors $\underline{V}_{m} ;$ complex magnetic flux phasor $\underline{\Phi}$

\section{Electromagnetic Coupling}

\subsection{Single Phase Electromagnetic Coupling}

The coupling of fundamental wave magnetic flux and magnetic potential (difference) interacting with instantaneous voltages and currents is elementary modeled in the electro magnetic coupling models SinglePhaseElectromagneticConverter . Figure 1 shows the magnetic phasors and a winding of an equivalent two pole machine. In this case one magnetic pole covers a spatial angle equal to $\pi$. The displayed coil is skewed and coil span is smaller than $\pi$. The coil can actually also be seen as a distributed winding. Skewing and distributed windings with respect to the fundamental wave are considered by the effective number of turns, represented by the parameter effectiveturns. The effective number of turns of a real machine is determined by the real number of turns, multiplied by the skewing factor and the chording factor. A more detail investigation on common windings and the determination of winding factors is published in $[10,18]$. A current through the investigated windings gives rise to magnetic potential (difference) distribution which magnitude is equal effective number of turns times the current. The peak of accessory sinusoidal magnetic potential (difference) caused by the current is in line with the orientation of the winding axis.

In an electric machine several windings of stator and rotor windings and permanent magnets contribute the total magnetic potential difference - depending on the type of machine. In the electromagnetic coupling model two physical laws are implemented, i.e., Ampere's law and the induction law.

\subsection{Ampere's Law}

Ampere's law states that the total exciting magneto motive force is equal to the magnetic potential difference. For the investigated single phase winding it is useful to define the complex number of turns:

final parameter Complex

$\mathrm{N}=$ effectiveTurns $\star$ Modelica. ComplexMath .exp (
Complex(0, orientation))

"Complex number of turns";

This complex quantity has the magnitude of the effective number of turns and the phase angle orientation. The magnetic potential (difference) of the coupling model, v_m, and the current of the investigated winding, i, are then related by:

\section{$V \_m=(2.0 / p i) * N * i$;}

The factor $2.0 / \mathrm{pi}$ is the consequence of averaging the sinusoidal fundamental wave flux waveform over one pole pair.

\subsection{Induction Law}

Induction law describes the the relationship between the time derivative of the magnetic flux and the induced voltage of the investigated winding. The projection of the complex magnetic flux onto the orientation times the effective number of turns is equal to the negative terminal voltage.

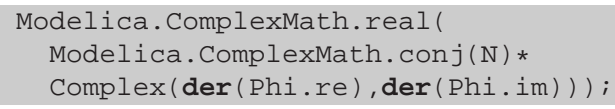

\subsection{Multi Phase Electromagnetic Coupling}

The multi phase electromagnetic coupling model is composed of a vector of $m$ single phase electromagnetic coupling models, where $m$ is the number of phases. The $m$ electrical pins of the single phase electromagnetic coupling model are connected to the m pins of the electrical multi phases connector used by the multi phase coupling model.

The $m$ magnetic fundamental wave ports of the single phase electromagnetic coupling models are connected in series. The magnetic series connection is a consequence of, first, each winding being exposed to the same magnetic flux wave - but being located spatially on different locations. Second, the total magnetic potential (difference) excited by all windings is determined by the sum of the magnetic potential (differences) of all individual windings.

\section{Phase Orientations - Winding Axes}

In the FundamentalWave library only symmetrical $m$ phase windings are considered. For multi phase systems and windings with phase numbers greater than three two different cases are distinguished, first, the number of phases is divisible by three and second, the number of phases is not divisible by three. The general function symmetricorientation for determining the orientations of windings of an $m$ phase electric machine is located in the package Modelica.Electrical. Multiphase.Functions:

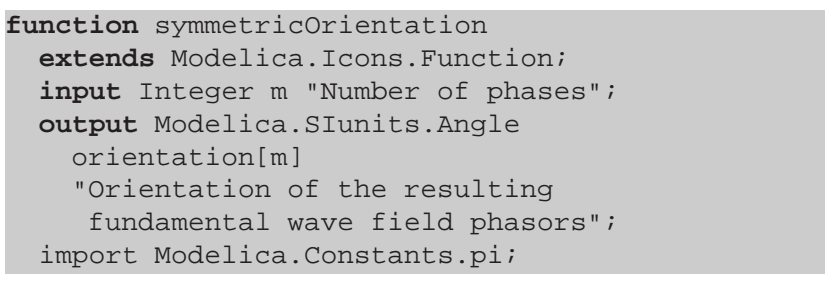




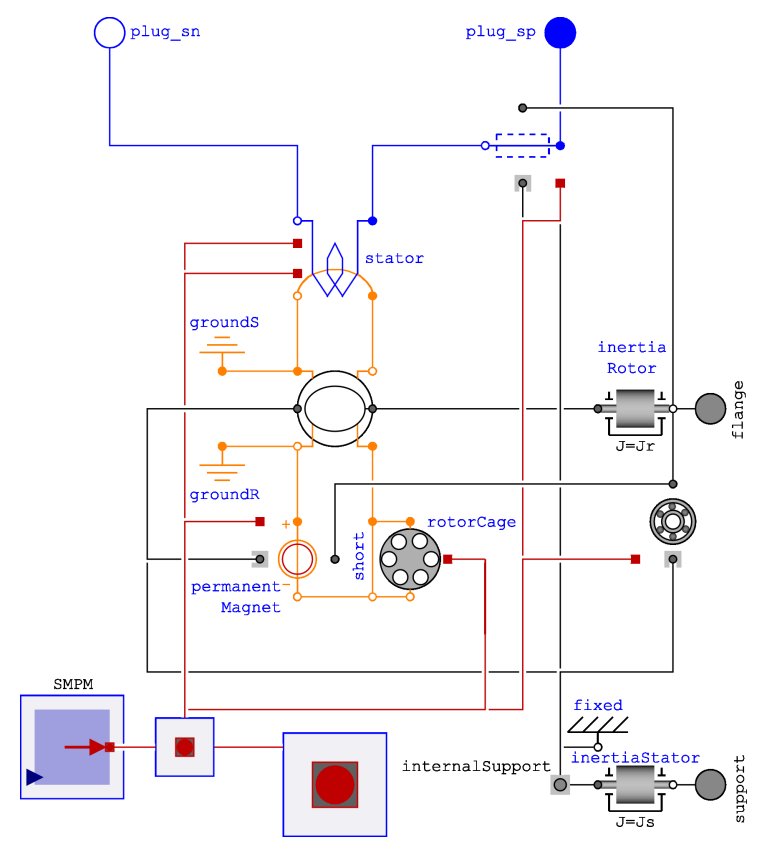

Figure 2: Permanent magnet synchronous machine with optional damper cage

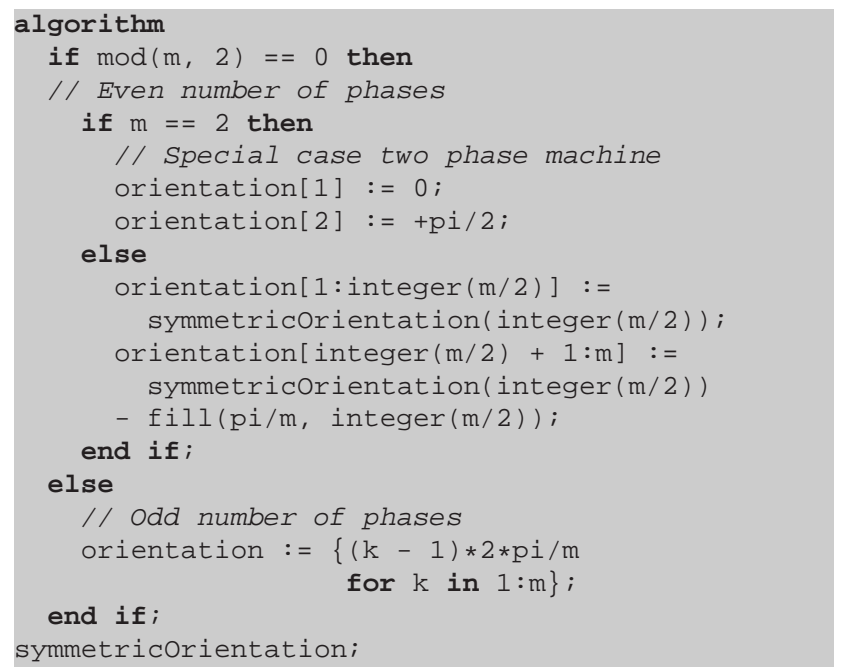

So the function is designed recursively so that subsystems are modularly designed. In the following some examples of phase numbers will be discussed. Two, four, eight, sixteen, thirty-two, etc. phase windings are currently not supported, as in general phase numbers equal to $2^{n}$ with integer $n$ are not considered.

In order to summarize mathematical equations for different phase numbers $m$ in the following, abbreviation orientation ${ }_{k}$ will be used to indicate the angles of the orientation of the winding axes of a symmetrical $m$ phase winding. So orientation $k_{k}$ is the $k$-th element (phase index) of the result vector returned by function symmetricorientation, called with argument $m$.

The symmetrically supply voltages and currents, respectively, have the phase angles

$$
\phi_{k}=\text {-orientation }{ }_{k}
$$

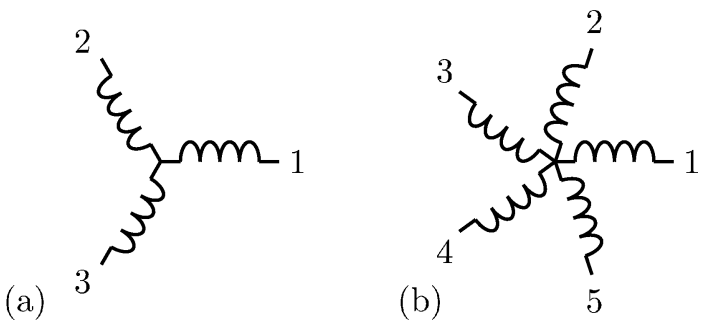

Figure 3: Winding axes of symmetrical (a) three phase winding and (b) five phase winding

(a)

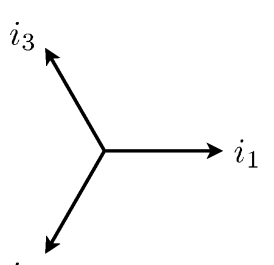

$i_{2}$

(b)

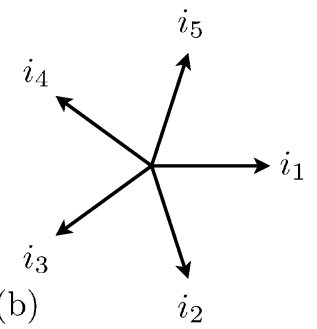

Figure 4: Symmetrical phase angles of voltages and currents, respectively, of (a) three phase winding and (b) five phase winding

The winding orientations and the phase shifts of the supplying system have the same magnitudes for each phase index $k$, but different signs. This is a general property of symmetrical systems supplying symmetrical windings, see Figs. 36.

In the following only symmetrical winding axes will be assumed. The phase angles of symmetrical voltage and current supply are also presented, even though symmetric supply is not assumed in the FundamentalWave library.

\subsection{Odd Phase Numbers}

For all odd phase numbers $m$ (not divisible by 2 ) the symmetrical orientations of the winding axes are

$$
\text { orientation }_{k}=\frac{2 \pi(k-1)}{m} \text {. }
$$

So this applies for the case $m=3, m=5, m=7, m=9$, $m=11, m=13, m=15$, etc., see Fig. 3-4.

\subsection{Even Phase Numbers}

For even phase numbers unequal to $2^{n}$ with integer $n$, the $m$ phase system is separated into two subsystems with $m / 2$ phases. The winding orientations of the second sub system lags the first sub system by $\frac{\pi}{m}$. This is then the point where the recursive determination of phase angle is initiated. For each of the two sub systems functions symmetricorientation is called, considering the lag angle $\frac{\pi}{m}$. It is important to explicitly note that the phase shift between the two sub systems is not $\frac{2 \pi}{m}$, since in this case the 

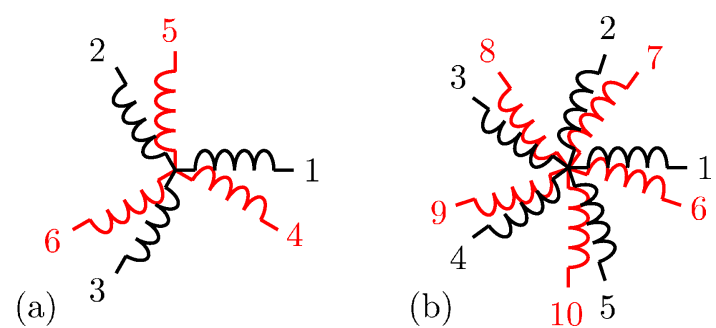

Figure 5: Winding axes of symmetrical (a) six phase winding and (b) ten phase winding
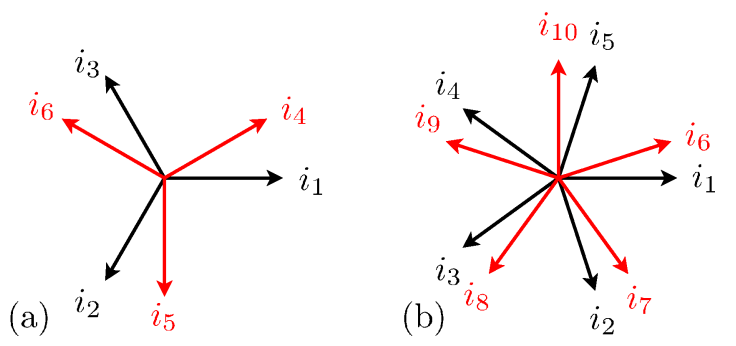

Figure 6: Symmetrical phase angles of voltages and currents, respectively, of (a) six phase winding and (b) ten phase winding

two sub systems where aligned at $\pm \pi$ which does not make sense in a technical system for redundancy reasons.

A six phase system is then separated into two three phase systems. The winding orientations of the second sub systems lags the first sub system by $\pi / 6$, see Figs. 5-6. The phase sequences (1-2-3) and (4-5-6) of the two sub systems are equal.

A ten phase system consists of two five phase systems with the phase sequences (1-2-3-4-5) and (6-7-8-9-10). The second sub systems lags the first sub system by $\frac{\pi}{10}$, see Figs. 56.

\subsection{Phase Numbers Divisible by Three}

Phase numbers divisible by three are either covered by subsection 4.1 and 4.2. Therefore, from a formal point of view no additional explanations are required to handle, for example, nine phase machines. Yet, a typical engineering approach and function symmetricorientation for numbering the phase numbers are different and may require some additional comments:

Practically, in most technical cases, electrical machines with phase numbers divisible by three will be supplied by an appropriate number of three phase inverters. For six phase systems this has already been demonstrated in subsection 4.2. In the FundamentalWave library nine phase systems are handled differently only in that sense, the sequence numbering the phase windings is most likely different from an engineer who uses three three phase inverters. In the engineering phases (1-2-3) are most likely assigned to the first inverter, phases (4-5-6) are assigned to the second inverter and phases (7-8-9) are assigned to the third inverters, see Fig. 7(a). In the FundamentalWave library the phase are
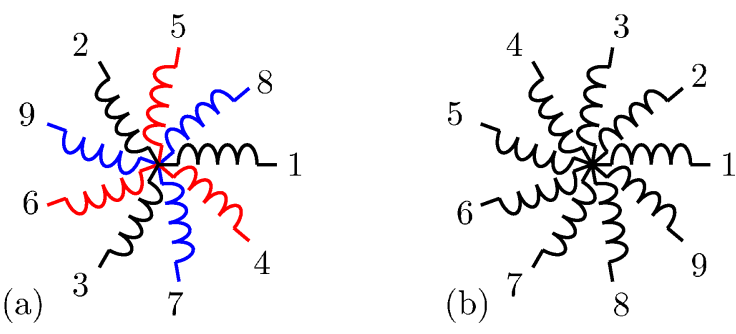

Figure 7: Numbering of nine phase symmetrical winding according to (a) an engineering approach using three three phase inverters and (b) the scheme of the FundamentalWave library

numbered according to Fig. 7(b). Even though the numbering is different the angles of the orientations are fully identical.

For a fifteen phase a design engineer could always argue whether such system can be seen as five three phase systems or as three five phase systems. However, from operating conditions point of view, there is no difference between these two cases. The numbering scheme of the FundamentalWave follows a formal scheme and the user decides how the machine phases are supplied.

\section{Magnetic Components}

All the existing magnetic components of the FundamentalWave library can be re-used for the multi-phase machine models, since the magnetic port representation did not change. In the current implementation only linear magnetic materials are considered. Saturation effects are not taken into account.

In all electric machine models of the FundamentalWave library the total magnetic reluctance is concentrated in the air gap model. An example of permanent magnet synchronous machine with optional damper cage is displayed in Fig. 2. In the actual implementation of the FundamentalWave library the magnetic reluctances of the stator, rotor and air gap are not individually modeled. Even the linear characteristic of the permanent magnet is represent by the total air gap reluctance of the machine. The total reluctance takes the variable reluctance of the air gap length $\delta$ into account. A sketch of the air gap and the reciprocal function $1 / \delta$ are shown in Fig. 8.

The effect of variable magnetic reluctance due to the uneven shape of the air gap and the arrangement of magnets, respectively, is called saliency. The effect of saliency on fundamental wave forms is fully considered by unequal direct $(d)$ and quadrature $(q)$ axis reluctances. For rotor fixed magnetic flux Phi and total magnetic potential difference $\mathrm{V} \_\mathrm{m}$ the following relationship applies:

$(p i / 2.0) * V \_m . r e=P h i \cdot r e * R \_m \cdot d ;$

The $d$ and $q$ axis are, however, fixed with the rotor structure. Magnetic rotor excitation of synchronous machines is, however, always aligned with the $d$ axis. 


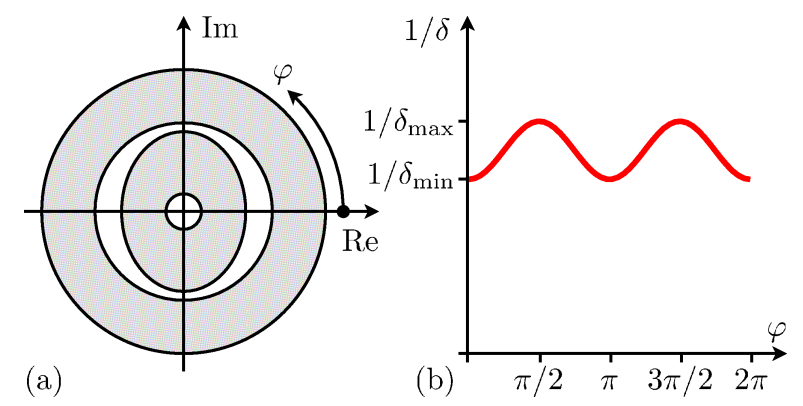

Figure 8: (a) Variable air gap length $\delta$ of a synchronous machine and (b) reciprocal air gap function $1 / \delta$ versus spatial angle $\varphi$ of an equivalent two pole machine

\begin{tabular}{r|l|l} 
Quantity & $m_{s}=3$ & \multicolumn{1}{|c}{$m_{s} \geq 3$} \\
\hline Nominal stator phase voltage & $V_{S N}^{\prime}$ & $V_{S N}=V_{s N}^{\prime}$ \\
Nominal stator phase current & $I_{S N}^{\prime}$ & $I_{S N}=I_{S N}^{\prime} \frac{3}{m}$ \\
Nominal stator frequency & $f_{S N}^{\prime}$ & $f_{S N}=f_{s N}^{\prime}$ \\
Nominal electrical torque & $\tau_{N}^{\prime}$ & $\tau_{N}=\tau_{N}^{\prime}$ \\
Nominal electrical stator power & $P_{S N}^{\prime}$ & $P_{S N}=P_{S N}^{\prime}$
\end{tabular}

Table 1: Parameters of machines with phases numbers equal and greater than three

In case that the saturation characteristics of the different regions of the machine shall be considered in the future, the magnetic equivalent circuit has to be adapted such way that each region is then represented by one non-linear reluctance.

\section{Parametrization}

Where do the parameters of a machine with $m_{s}$ stator phases come from? First, in the design stage of the machine, an engineer determines these parameters from finite element analysis or any other electromagnetic design software. Second, the parameters of a three phase machine are known or estimated and the users wants to determine the parameters of an equivalent $m$ phase machine. The equivalence then often refers to equivalent speed, frequency, torque, power, phase voltage, power factor and efficiency. For the second case the exact calculations will be provided in the following:

Assume, the nominal parameter of a three phase and arbitrary $m$ phase machine as listed in Tab. 1 . All the parameters of a three phase machine are indicated with '. According to the relationship between the $m$ phase nominal phase voltage and current, all resistances and inductances of an $m$ phase machine are scaled with $m / 3$. A list of relevant parameters is summarized in Tab. 2.

The rotor winding of squirrel cage induction machines are implemented as equivalent $m_{s}$ phase windings - where $m_{s}$ is the number of stator phases. Slip ring induction machines may have different phases numbers of stator and rotor where $m_{r}$ is the number of rotor phases. For synchronous machines with permanent magnets, electrical excitation and reluctance rotor, the optional damper cage is implemented

\begin{tabular}{r|c|l} 
Quantity & $m=3$ & \multicolumn{1}{|c}{$m \geq 3$} \\
\hline Stator resistance & $R_{s}^{\prime}$ & $R_{s}=R_{s}^{\prime} \frac{m}{3}$ \\
Stator stray inductance & $L_{s \sigma}^{\prime}$ & $L_{s \sigma}=L_{s \sigma}^{\prime} \frac{m}{3}$ \\
Main field inductance & $L_{m}^{\prime}$ & $L_{m}=L_{m}^{\prime} \frac{m}{3}$ \\
Main field inductance, $d$-axis & $L_{m d}^{\prime}$ & $L_{m d}=L_{m d}^{\prime} \frac{m}{3}$ \\
Main field inductance, $q$-axis & $L_{m q}^{\prime}$ & $L_{m q}=L_{m q}^{\prime} \frac{m}{3}$
\end{tabular}

Table 2: Stator parameters of three and $m_{s} \geq 3$ phase machines

\begin{tabular}{|c|c|c|}
\hline Quantity & $m=3$ & $m \geq 3$ \\
\hline \multicolumn{3}{|c|}{ Induction machine with squirrel cage } \\
\hline Rotor cage resistance & $R_{r}^{\prime}$ & $R_{r}=R_{r}^{\prime} \frac{m_{s}}{3}$ \\
\hline Rotor stray inductance & $L_{r \sigma}^{\prime}$ & $L_{r \sigma}=L_{r \sigma}^{\prime} \frac{m_{s}}{3}$ \\
\hline \multicolumn{3}{|c|}{ Induction machine with slip ring rotor } \\
\hline Rotor cage resistance & $R_{r}^{\prime}$ & $R_{r}=R_{r}^{\prime} \frac{m_{r}}{3}$ \\
\hline Rotor stray inductance & $L_{r \sigma}^{\prime}$ & $L_{r \sigma}=L_{r \sigma}^{\prime} \frac{m_{r}}{3}$ \\
\hline \multicolumn{3}{|l|}{ All synchronous machines } \\
\hline Damper cage resistance, $d$-axis & $R_{r d}^{\prime}$ & $R_{r d}=R_{r d}^{\prime}$ \\
\hline Damper cage resistance, $q$-axis & $R_{r q}^{\prime \prime}$ & $R_{r q}=R_{r q}^{\prime}$ \\
\hline Damper cage & & \\
\hline stray inductance, $d$-axis & $L_{r \sigma, d}^{\prime}$ & $L_{r \sigma, d}=L_{r \sigma, d}^{\prime}$ \\
\hline Damper cage & & \\
\hline stray inductance, $q$-axis & $L_{r \sigma, q}^{\prime}$ & $L_{r \sigma, q}=L_{r \sigma, q}^{\prime}$ \\
\hline
\end{tabular}

Table 3: Rotor parameters of machines with three and $m_{s} \geq$ 3 and $m_{r} \geq 3$ phases

with salient rotor parameters with respect to the direct $(d)$ and quadrature $(q)$ axis. So there is no difference between three and multi phase damper cage models and parameters. The rotor cage parameters of all machine types are summarized in Tab. 3.

\section{Examples}

In the FundamentalWave library there are examples for all types of machines, comparing three phase and multi phase $(m=5)$ machines. The three and five phase machines are operated with equal nominal phase voltages. The parameters of the five phase machine are parameterized such way automatically that the phase number can be increased without changing torques and powers for the multi phase machines. In a duplicate example the phase numbers can be changed for from five to higher numbers.

The following examples are included in the FundamentalWave library:

\subsection{Induction Machine with Squirrel Cage}

In model Examples.AIMC_DOL_Multiphase two asynchronous induction machines with squirrel cage rotor are started directly on line (DOL) by means of an ideal switch; see Figure 9. The machines start from standstill. 


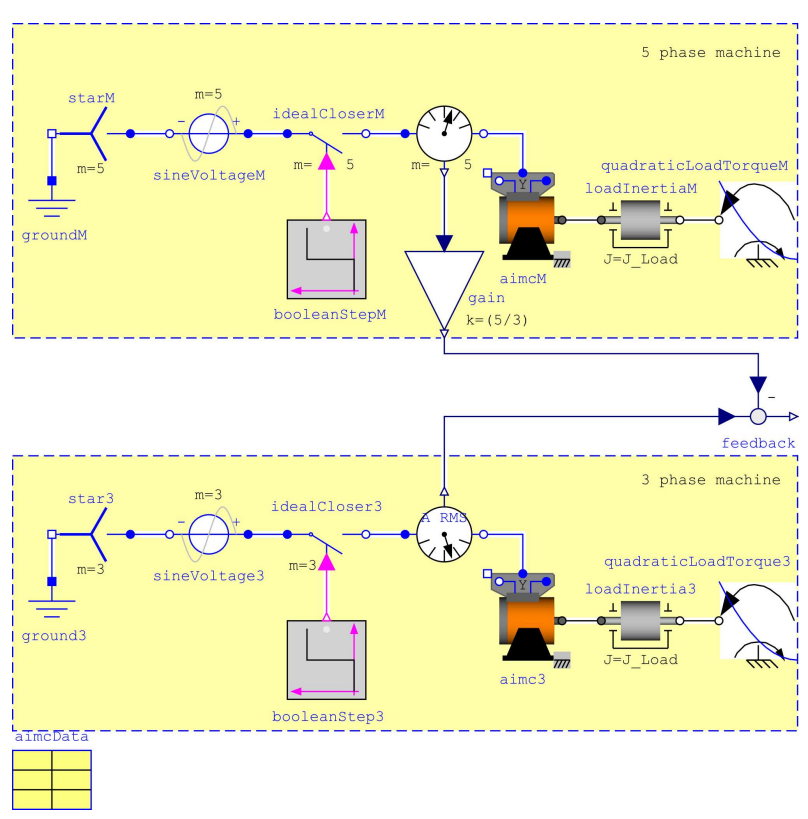

Figure 9: Comparing a three and a multi phase $(m=5)$ permanent magnet synchronous machine, operated on an idealized voltage inverter

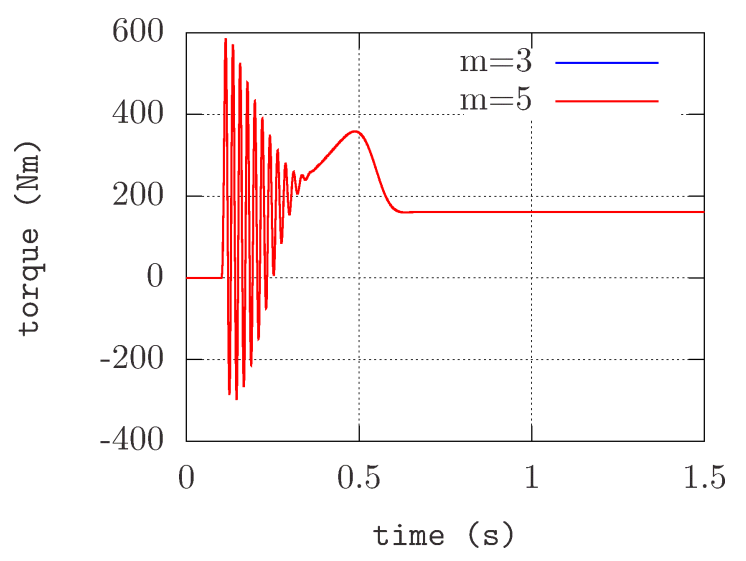

Figure 10: Simulation result of electrical torque of a three and five phase squirrel cage induction machines; the torques are equal

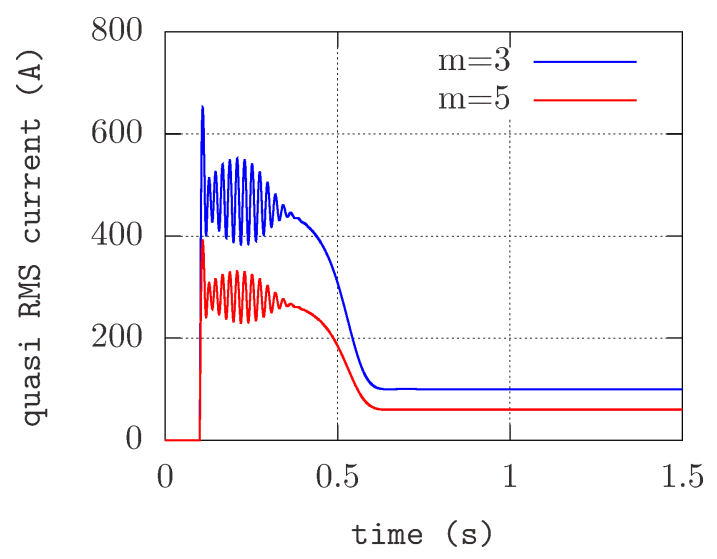

Figure 11: Simulation result of the quasi RMS currents of a three and five phase squirrel cage induction machines; the current ratio is five to three

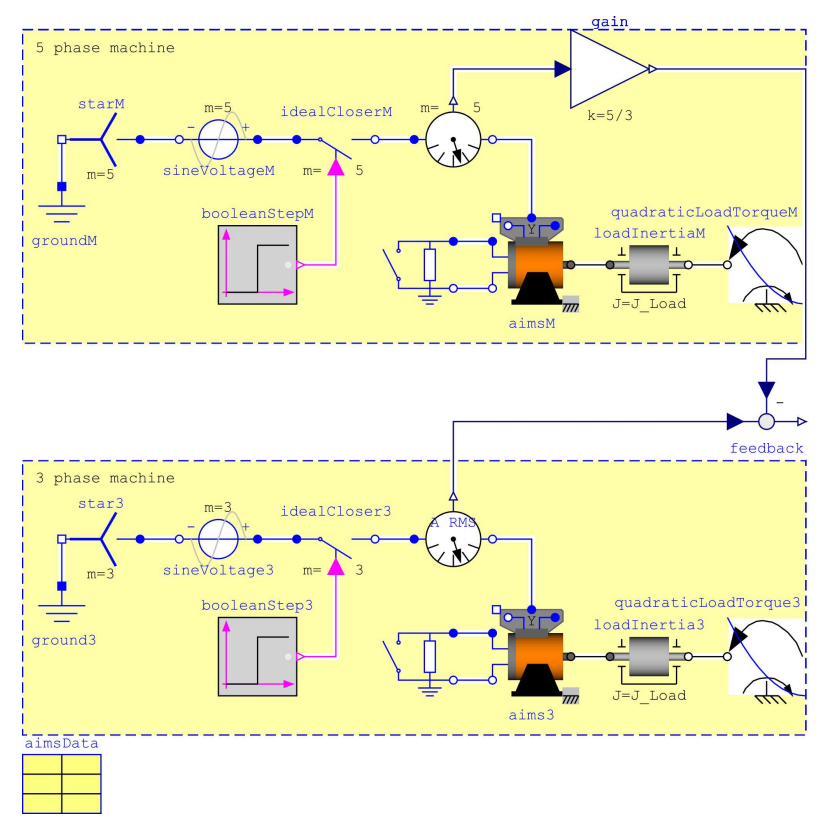

Figure 12: Comparing a three and a multi phase $(m=5)$ induction machine started directly on line, operated on an idealized voltage inverter

The mechanical load is modeled by means of a quadratic speed dependent load torque and an additional load inertia. This example demonstrates equivalent dynamic behavior of the three and five phase machine. Particularly, the electrical torque, speed, and the particular losses are equal.

Both machines have the same nominal phase voltage, but different nominal phase currents according to Tab. 1. In Fig. 10 the two identical electric torques of the two machines are shown. The different quasi RMS currents of two machines are displayed in Fig. 11. The current ratio is equal to five over three.

\subsection{Induction Machine with Slip Ring Rotor}

Model Examples.BasicMachines.AIMS_Start_MultiPhase compares a three and a five phase slip ring induction machine, operating the stator direct on line; see Fig. 12 The number of stator phases $m_{s}=5$ and the number of rotor phases, $m_{r}=5$, are equal. The multi phase rotor windings of each machine are connected with a rheostat which is shorted after a give time period tRheostat $=$ 1.0 second. The rheostat enables a greater starting torque - but worse efficiency. Therefore, after one second, the rheostats are shortened to achieve a higher efficiency and speed of the machines. The user can copy the example and change the rotor phase number $m_{r}$ such way that it differs from the stator phase number, $m_{s}$. This case is also supported the FundamentalWave library. In Fig. 13 and 14 the electromagnetic torques and the quasi RMS currents of the two machines are compared. The torques are identical and the current ratio is five to three according to Tab. 1. 


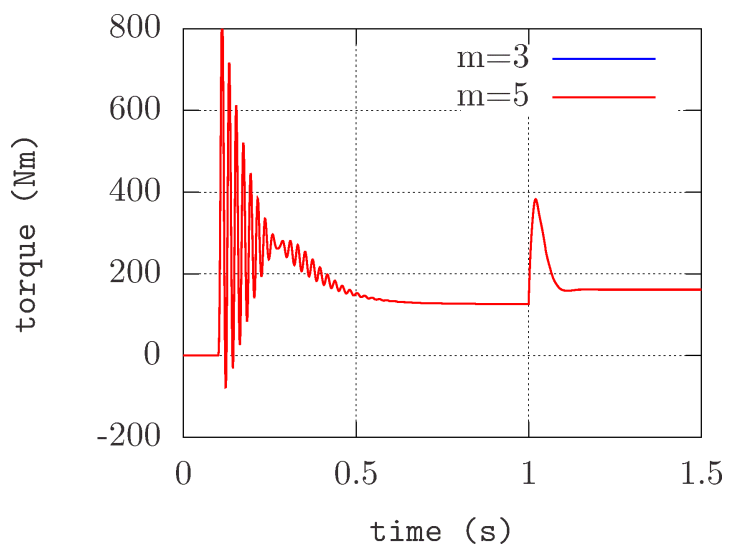

Figure 13: Simulation result of electrical torque of a three and five phase slip ring induction machines; the torques are equal

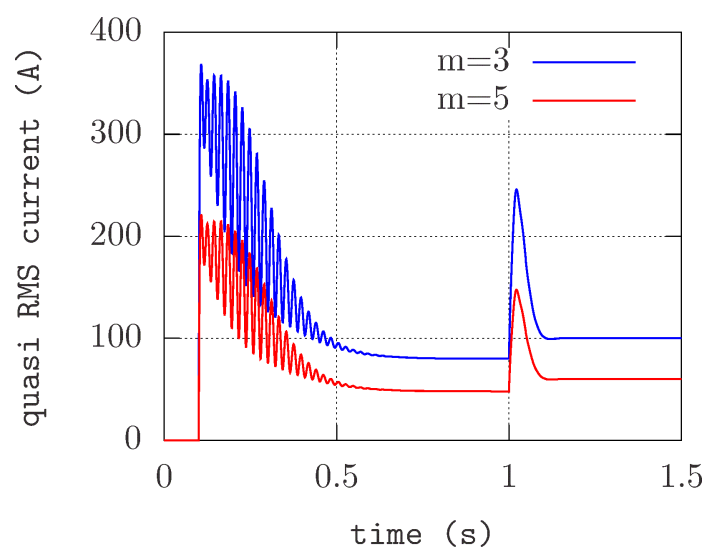

Figure 14: Simulation result of the quasi RMS currents of a three and five phase slip ring induction machines; the current ratio is five to three

\subsection{Synchronous Generator with Electrical Excitation}

In example Examples.SMPM_Generator two mains supplied electrical excited synchronous machine with three and five stator phases are compared; see Fig. 16. For each machine shaft speed is constant and slightly different than synchronous speed. In this experiment each rotor is forced to make a full revolution relative to the magnetic field. In Fig. 16 the generated torques versus load angle are shown for a fixed level of excitation. In addition to the sinusoidal waveform of the torque a second harmonic component is superimposed due to the saliency of the rotor. However, for the investigated machines, the saliency effect is very small so that the torque waveform almost appears as a pure sine wave. The quasi RMS currents of the two machines are compared in Fig. 17. The current ratios of the three and five phase machine is five to three.
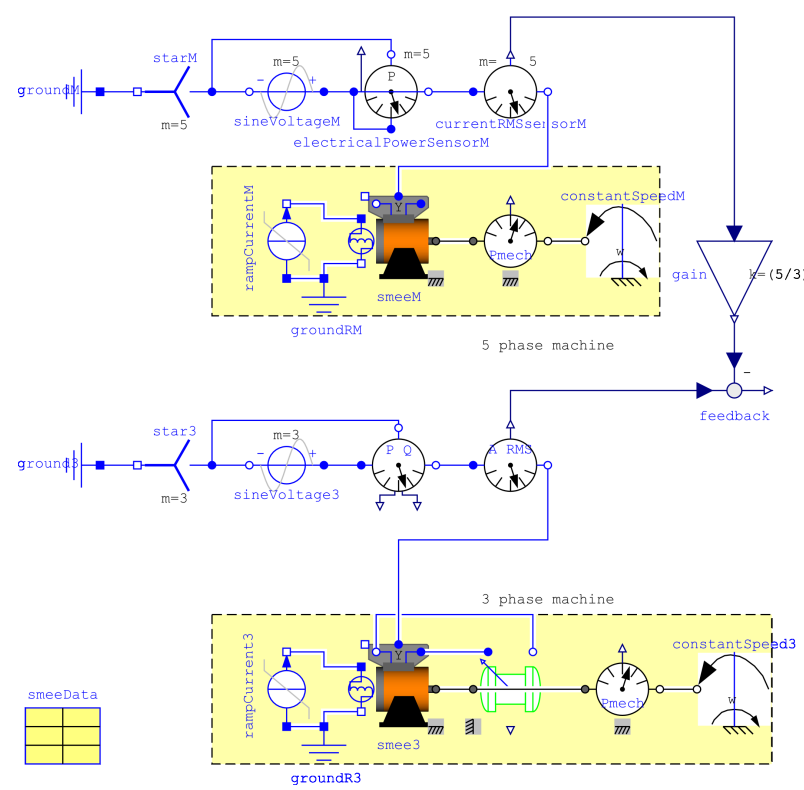

Figure 15: Comparing a three and a multi phase $(m=5)$ permanent magnet synchronous machine, operated on an idealized voltage inverter

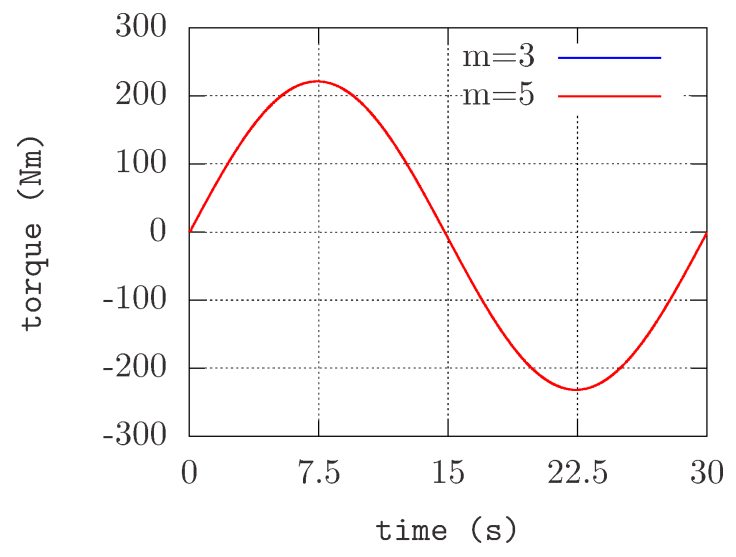

Figure 16: Simulation result of electrical torque, comparing a three and five phase

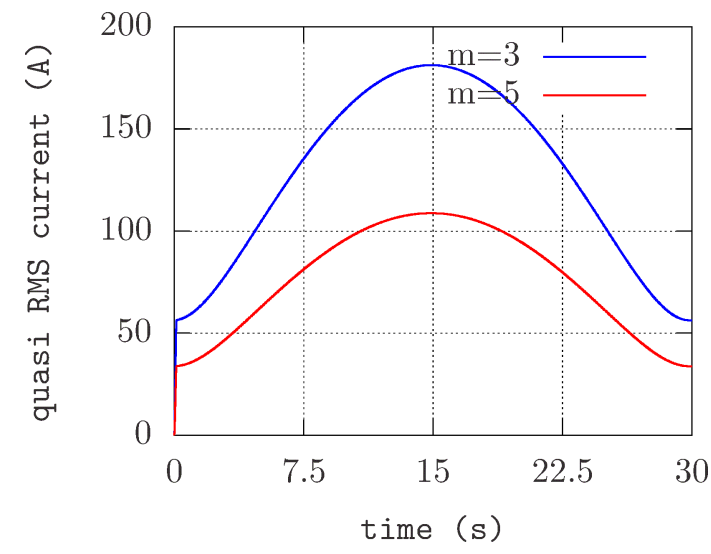

Figure 17: Simulation result of the quasi RMS currents of a three and five phase synchronous machine with electric excitation; the current ratio is five to three 


\section{Conclusions}

The paper presents an extension of the FundamentalWave library towards multi phase stator (and rotor) windings with phase numbers greater or equal than three. This library is included in the MSL 3.2.1. Assumptions and limitations of the presented implementation are explained. In the new FundamentalWave library only symmetrical windings are supported. The structures of symmetrical multi phase windings and supplies are introduced.

The parametrization of the multi phase machines is discussed. Conversion tables for parameterizing multi phase machines equivalent to three phase machines are presented. Simulation examples of three and equivalent five phase induction and synchronous machines are presented and compared.

\section{Acknowledgement}

The research leading to these results has received funding from the ENIAC Joint Undertaking under grant agreement no. 270693-2 and from the Österreichis- che Forschungsförderungsgesellschaft mbH under project no. 829420.

\section{References}

[1] B. Stumberger, G. Stumberger, A. Hamler, M. Trlep, M. Jesenik, and V. Gorican, "Increasing of output power capability in a six-phase flux-weakened permanent magnet synchronous motor with a third harmonic current injection," IEEE Transactions on Magnetics, vol. 39, pp. 3343-3345, 2003.

[2] D. G. Dorrell, C. Y. Leong, and R. A. McMahon, "Analysis and performance assessment of six-pulse inverter-fed three-phase and six-phase induction machines," IEEE Transactions on Industry Applications, vol. 42, pp. 1487-1495, November/December 2006.

[3] G. Aroquiadassou, A. Mpanda-Mabwe, F. Betin, and G.-A. Capolino, "Six-phase induction machine drive model for fault-tolerant operation," SDEMPED, 2009.

[4] H. A. Toliyat, M. M. Rahimian, and T. A. Lipo, “dq modeling of five phase synchronous reluctance machines including third harmonic of air-gap mmf," Conference Record of the 1991 IEEE Industry Applications Society Annual Meeting, 1991., pp. 231-237, 1991.

[5] D. Dujic, M. Jones, and E. Levi, "Features of two multi-motor drive schemes supplied from fivephase/five-leg voltage source inverters," Conference Proceedings on Power Conversion and Intelligent Motion, PCIM, Nuremberg, Germany, no. S2d-2, 2008.

[6] D. Dujic, M. Jones, and E. Levi, "Analysis of output current ripple rms in multiphase drives using space vector approach," IEEE Transactions on Power Electronics, vol. 24, no. 8, pp. 1926-1938, 2009.
[7] R. L. A. Ribeiro, C. B. Jacobina, A. M. N. Lima, and E. R. C. da Silva, "A strategy for improving reliability of motor drive systems using a four-leg three-phase converter," Sixteenth Annual IEEE Applied Power Electronic Conference and Expoition. APEC 2001, vol. 1, pp. 385-391, 2001.

[8] R. Errabelli and P. Mutschler, "Fault-tolerant voltage source inverter for permanent magnet drives," IEEE Transactions on Power Electronics, vol. 27, pp. 500 -508 , feb. 2012.

[9] T. Treichl, Regelung von sechssträngigen permanenterregten Synchronmaschinen für den mobilen Anwendungsfall. PhD thesis, FernUniversität, 2006.

[10] C. Kral and A. Haumer, "Modelica libraries for DC machines, three phase and polyphase machines," International Modelica Conference, 4th, Hamburg, Germany, pp. 549-558, 2005.

[11] C. Kral and A. Haumer, Object Oriented Modeling of Rotating Electrical Machines. INTECH, 2011.

[12] C. Kral and A. Haumer, "The new fundamentalwave library for modeling rotating electrical three phase machines," 8th International Modelica Conference, 2011.

[13] T. A. Lipo, "A d-q model for six phase induction machines," Conference Record of International Conference on Electrical Machines, ICEM, pp. 860-867, 1980.

[14] E. Andresen and K. Bieniek, "Der Asynchronmotor mit drei und sechs Wirkungssträngen am stromeinprägenden Wechselrichter," Archiv für Elektrotechnik, vol. 63, pp. 153-167, 1981.

[15] H.-H. Jahn and R. Kasper, "Koordinatentransformationen zur Behandlung von Mehrphasensystemen," Archiv für Elektrotechnik, vol. 56, pp. 105-111, 1974.

[16] J. Stepina, Einführung in die allgemeine RaumzeigerTheorie der elektrischen Maschinen. Kaiserslautern: Vorlesungsskriptum, Universität Kaiserslautern, 1979.

[17] C. Kral, A. Haumer, M. Bogomolov, and E. Lomonova, "Harmonic wave model of a permanent magnet synchronous machine for modeling partial demagnetization under short circuit conditions," XXth International Conference on Electrical Machines (ICEM), pp. 295 -301, Sept. 2012.

[18] C. Kral and A. Haumer, "Simulation of electrical rotor asymmetries in squirrel cage induction machines with the extendedmachines library," International Modelica Conference, 6th, Bielefeld, Germany, no. ID140, pp. 351-359, 2008. 